\title{
Aspergillus Section Nigri in Onions
}

\author{
Rachel Bertoldo (I), Marta Hiromi Taniwaki (I), Maria Helena \\ Pelegrinelli Fungaro (II), Beatriz Thie lamanaka (I)
}

(I) ITAL - Instituto de Tecnologia de Alimentos (Av. Brasil, 2880 - CEP 130.70-178 CampinasSP, Brasil), (II) UEL - Universidade Estadual de Londrina (Rodovia Celso Garcia Cid, Km 380 -

Campus Universitário, CEP 86057-970, Londrina)

\section{Resumo}

Various fungal diseases can spoil onions in the field and post-harvest and may persist during transportation, storage and marketing. In Brazil, annual losses can reach $50 \%$. One of the diseases is "black mold", related to black Aspergillus, which is commonly found in foods. Some species of Aspergillus section Nigri are capable of producing mycotoxins, such as ochratoxin A. Ochratoxin A is considered to be nephrotoxic, teratogenic and immunotoxic. In onions, the presence of black Aspergillus has been reported as being responsible for the disease "black rot", but there are no reports on the identification of these species. The aim of this work was to isolate and identify Aspergillus section Nigri species producing ochratoxin A in onions. Nineteen samples from the state of São Paulo were collected. For the isolation of fungi, direct plating on agar Dichloran 18\% Glycerol (DG18) was used after disinfection with sodium hypochlorite. The toxigenic potential of the species was tested on yeast extract sucrose (YESA), extraction of the toxin by the agar plug technique and detection by thin layer chromatography (TLC). Ochratoxin A in onion samples was extracted with methanol and subjected to the cleaning step in an immunoaffinity column. The detection and quantification were performed by liquid chromatography of high efficiency with fluorescence detection. The average infection by Aspergillus section Nigri was 70\%, varying from $22 \%$ to $100 \%$, with a total of 658 isolates. Only 4 isolates were ochratoxin A producers. The onion samples were not contaminated with ochratoxin A. We conclude that onions have a high fungal contamination by these 
species, but low production and occurrence of ochratoxin A. The morphological evaluation and molecular testing of the strains are ongoing in order to confirm the Aspergillus section Nigri identity.

Palavras-Chave: Fungi, Ochratoxin A, Onion Agência de Fomento: FAPESP 